\title{
Autoimmune diseases associated with autoimmune hepatitis in a series of 21 cases
}

\author{
Bennani GK*, Benelbarhdadi I, Bourehma M, Berhili C, Lagdali N and Ajana FZ \\ Department of Diseases of the Digestive System, Medicine C, CHU IBN SINA- Souissi Med University V-Rabat, Morocco
}

\begin{abstract}
Autoimmune hepatitis (HAI) is a chronic inflammatory disease of the liver of dysimmune origin which is known by its coexistence with other autoimmune manifestations. The aim of our work is to determine the prevalence and the type of disorders HAI-associated auto-immune systems. Our study is focused on 21 consecutive cases of autoimmune hepatitis diagnosed in our department, Among the $86 \%$ were women, 18 out of 21 patients (78\%) had an autoimmune disease associated with HAI Associated autoimmune diseases were predominant over autoimmune dysthyroiditis and overlap syndrome The treatment was mainly based on a combined therapy (corticosteroid and immural) combined with urso deoxycholic acid if overlapping syndrome with specific treatment of the associated autoimmune disease.
\end{abstract}

\section{Introduction}

Autoimmune hepatitis (HAI) is a chronic inflammatory disease of the liver of dysimmune origin with spontaneous cirrhogenic evolution. The coexistence of autoimmune manifestations testifies to the existence of a predisposing genetic terrain.

\section{Aim of study}

The goal of our work is to determine the prevalence and type of autoimmune disorders associated with HAI.

\section{Patients and methods}

Mono-centric retrospective study of 21 consecutive cases of autoimmune hepatitis diagnosed in our department, over an 8-year period between January 2011 and July 2019.

All our patients are followed in a specialized consultation of HAI The diagnosis was only made after excluding other causes of chronic liver disease: viral B or C, drug, alcoholic, metabolic and genetic.

In fact, serologies for viral hepatitis B and $\mathrm{C}$ were carried out in all cases. The toxic and medicinal causes were eliminated on anamnestic criteria. There were 16 cases of isolated HAI and 5 cases of overlap syndrome (OS). The score used for the diagnosis of HAI is below the diagnosis of overlap syndrome was made when at least two criteria of HAI were combined with at least two other criteria of CBP:

Criteria for HAI: ALT $>5 \times$ ULN - IgG $>2 \times$ ULN

1. Presence of anti-smooth muscle antibodies (AML) $\geq 1 / 80$

2. Hepatitis of moderate or severe interface, this criterion being mandatory

CBP criteria: Unexplained cholestasis: alkaline phosphatases (PAL) $>1.5 \times$ ULN

1. or gamma-glutamyltransferase (GGT) $>3 \times \mathrm{ULN}$

2. Presence of anti-mitochondria type 2 antibodies (AMA2) $\geq 1 / 40$.
After the diagnosis of HAI, the search for the other most frequently associated autoimmune diseases is systematic.

\section{Results}

\section{Age and gender}

There were 18 women (86\%) and 3 men (14\%) with a sex ratio of 0.16 . The age at diagnosis was 39.4 years (16-76 years) on average.

\section{Telltale signs}

The condition was revealed by jaundice in $47 \%$, asthenia in 3 cases $60 \%$, isolated pruritus in $21.2 \%$ of patients and pain in the right hypochondrium in $21.1 \%$.

\section{Biological signs}

Liver biology was characterized by the presence of a mixed syndrome combining cytolysis and cholestasis in 18 cases $(86 \%)$. The level of transaminases was higher than $10 \mathrm{x}$ ULN in 4 cases (21.1\%), superior to $5 \mathrm{x}$ ULN in 5 cases (26.3\%). A hypergammaglobulinemia carrying on IgG was found in 14 cases (66\%). A biological syndrome of hepatocellular insufficiency was already present at the time of diagnosis in patients $(38 \%)$. Moderate pancytopenia, related to hypersplenism, was observed in 3 patients (14.2\%).

\section{Associated auto immune disease}

18 out of 21 patients $(78 \%)$ had autoimmune disease associated with HAI. $62.5 \%$ had a single associated autoimmune disease, $19 \%$

${ }^{\star}$ Correspondence to: Ghita Bennani, Department of Diseases of the Digestive System, Medicine C, CHU IBN SINA-Souissi Med University, V-Rabat, Morocco, Tel: +212 5376-76464; E-mail: ghita_med5@yahoo.fr

key words: auto immune hepatitis, related autoimmune disease, overlap syndrome, autoimmune thyroiditis

Received: November 04, 2019; Accepted: November 18, 2019; Published: November 21, 2019 
had two associated autoimmune diseases. The diagnosis of associated autoimmune diseases was concomitant with that of HAI in 13 cases (72.2\%), preceded that of HAI in 2 cases $(11.1 \%)$ and 3 cases $(16.6 \%)$ were diagnosed at follow-up. of the HAI. The associated autoimmune diseases were; autoimmune dysthyroiditis $(n=9)$, CBP-HAI overlap syndrome $(n=5)$, CSP-HAI $(n=1)$, dry syndrome $(n=3)$, rheumatoid arthritis $(n=1)$, insulin-dependent diabetes $(n=1)$

\section{Discussion}

\section{Age}

HAI is a condition seen particularly in young adults. The first manifestations of HAI can be observed between the age of 1 year and the 8th decade. A study carried out in France concluded that the average age at diagnosis was 43 years with extremes of age which varied between 16 years and 75 years (1), another study in Algeria had found an average age of 38 years with age extremes ranging from 17 years to 73 years. (2) In our series, the age of diagnosis agrees with the results of other studies and varies between 16 years and 74 years with an average of 39.4 years (Table 1 ).

\section{Gender}

HAI is mainly a disease of young women but its incidence in the male sex is increasing, in our study as in the others the sex ratio is 4 $\mathrm{F} / 1 \mathrm{H}[1-3]$ (Table 2).

\section{Mode of clinical presentation}

HAI can be in the form of acute or chronic liver disease with a wide variety of clinical presentations ranging from asymptomatic to fulminant hepatitis.

In our study $64 \%$ presented in a Table of chronic liver disease $22 \%$ in a table of acute hepatitis and in $14 \%$ of cases, the patients were asymptomatic. In the latter case, the diagnosis is evoked either before a disturbance of the hepatic balance or anomaly on the clinical examination. The HAI produces often heterogeneous tables of varying severity (Table 3 ).

\section{Autoimmune diseases associated with HAI}

HAI can be associated with other autoimmune diseases as

Table 1. Comparison of the age of diagnosis

\begin{tabular}{|c|c|c|c|}
\hline Series & Number of cases & Average age & Extremes of age \\
\hline Hakem et al. [2] & 50 & $38 \mathrm{Y}$ & $17-73 \mathrm{Y}$ \\
\hline Nguyen et al. [1] & 23 & $43 \mathrm{Y}$ & $16-75 \mathrm{Y}$ \\
\hline Our series & 21 & $39 \mathrm{Y}$ & $16-74 \mathrm{Y}$ \\
\hline
\end{tabular}

Table 2. Comparison of the gender of diagnosis

\begin{tabular}{|c|c|c|c|}
\hline Series & Number of cases & $\begin{array}{c}\text { Percentage of } \\
\text { women }\end{array}$ & Sex - ratio \\
\hline Kchir et al. [3] & 50 & 27 & $54 \%$ \\
\hline Nguyen et al. [1] & 23 & 19 & $82.6 \%$ \\
\hline Notre series & 21 & 18 & $86 \%$ \\
\hline
\end{tabular}

Table 3. Clinical manifestations according to the different studies

\begin{tabular}{|c|c|c|c|c|c|}
\hline series & jaundice & asthenia & $\begin{array}{l}\text { Pain of the right } \\
\text { hypochondrium }\end{array}$ & pruritus & Hepatomegaly \\
\hline Chaouali & $80 \%$ & $66 \%$ & $40 \%$ & $43 \%$ & $33 \%$ \\
\hline Hakem & $72 \%$ & $96 \%$ & $24 \%$ & $22 \%$ & $56 \%$ \\
\hline Burgart & $80 \%$ & $85 \%$ & $50 \%$ & - & $80 \%$ \\
\hline Our series & $47 \%$ & $68 \%$ & $21.1 \%$ & $21.1 \%$ & $63 \%$ \\
\hline
\end{tabular}

Table 4. Comparison of autoimmune diseases associated with HAI between series

\begin{tabular}{|l|c|c|c|c|}
\hline The associated autoimmune diseases & Gourdas & Nguyen & Chaouali & Notre series \\
\hline autoimmune thyroiditis & 7.8 & $17.4 \%$ & & $42 \%$ \\
\hline insulin-dependent diabetes & $10.5 \%$ & $4.35 \%$ & & \\
\hline Syndrome sec & & & $13.3 \%$ & \\
\hline Overlap syndrome & & $4.35 \%$ & $20 \%$ & $28 \%$ \\
\hline Polyarthritis rheumatoid & $5.22 \%$ & $4.35 \%$ & & $4.7 \%$ \\
\hline coeliac disease & & & $3.3 \%$ & \\
\hline
\end{tabular}

well as system diseases. This association has often been reported in the literature with a frequency varying from 26 to $40 \%$ such as autoimmune thyroiditis, celiac disease, Goujerot-Sjogren syndrome, rheumatoid arthritis and insulin-dependent diabetes. The coexistence of autoimmune manifestations testifies to the existence of a common predisposing genetic terrain thus constituting an important criterion for the diagnosis of this hepatopathy. In our study, $87 \%$ of patients had at least one autoimmune disease associated with HAI: $62.5 \%$ had a single associated autoimmune disease, 19\% had two associated autoimmune diseases.

The associated autoimmune diseases were; autoimmune dysthyroiditis $(n=9)$, CBP-HAI overlap syndrome $(n=5)$, CSP-HAI $(n=1)$, dry syndrome $(n=3)$, rheumatoid arthritis $(n=1)$, insulindependent diabetes $(n=1)$. The study by Debbeche et al. [9] showed that 27 patients $(31 \%)$ out of a total of 87 patients had at least one autoimmune disease associated with autoimmune hepatitis, mainly type 1 diabetes, auto-thyroiditis. immune system and Gougerot Sjögren's syndrome. A Tunisian study by Chaouali showed that 15 out of 30 patients had at least one autoimmune disease associated with liver disease, or $50 \%$ of the cases. These diseases were dominated by type 1 diabetes, primary biliary cirrhosis and autoimmune hemolytic anemia. The "CBP-HAI" overlap syndrome was retained in 6 patients, i.e. in $20 \%$ of the cases (Table 4).

\section{Conclusion}

HAI is frequently associated with other autoimmune diseases whose screening must be systematic. The associated autoimmune diseases mainly found in our series are overlap syndrome and autoimmune thyroiditis.

\section{References}

1. Nguyen Y, Hillaire S, Marroun I, Roumier M, Sené T, et al. (2010) Autoimmune hepatitis: monocentric series of 23 cases followed in internal medicine, France. Internal Med J: A52.

2. Hakem D, Berrah A, Berkane S, Asselah H, Aït-Younes S, et al. (2005) Autoimmune chronic active hepatitis: anatomoclinic's study of 50 patients. Internal Rev Med 26 : 858-865. [Crossref]

3. Kchir H, Maamouri N, Belkahla N, Ouerghi H, Ben F, et al. (2010) Autoimmune hepatitis in adults: about 32 cases, Tunisia. Internal Med J: S166-S167.

Copyright: (C2019 Bennani GK. This is an open-access article distributed under the terms of the Creative Commons Attribution License, which permits unrestricted use, distribution, and reproduction in any medium, provided the original author and source are credited. 